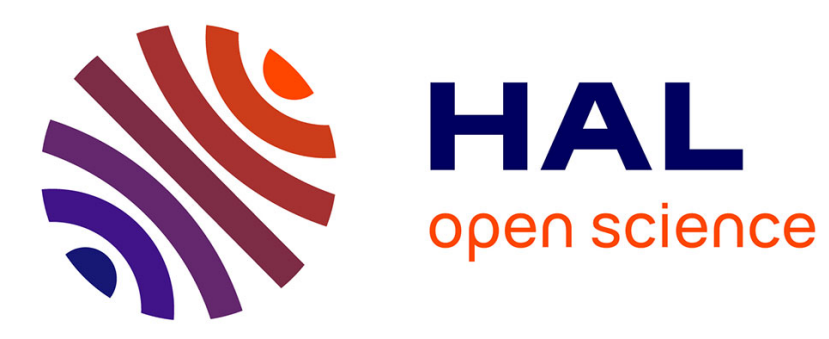

\title{
DNA directed immobilization glycocluster array: applications and perspectives.
}

Yann Chevolot, Emmanuelle Laurenceau, Magali Phaner-Goutorbe, Virginie

Monnier, Eliane Souteyrand, Albert Meyer, Thomas Gehin, Jean-Jacques Vasseur, François Morvan

\section{To cite this version:}

Yann Chevolot, Emmanuelle Laurenceau, Magali Phaner-Goutorbe, Virginie Monnier, Eliane Souteyrand, et al.. DNA directed immobilization glycocluster array: applications and perspectives.. Current Opinion in Chemical Biology, 2014, 18, pp.46-54. 10.1016/j.cbpa.2013.12.009 . hal00952596

\section{HAL Id: hal-00952596 https://hal.science/hal-00952596}

Submitted on 19 Apr 2019

HAL is a multi-disciplinary open access archive for the deposit and dissemination of scientific research documents, whether they are published or not. The documents may come from teaching and research institutions in France or abroad, or from public or private research centers.
L'archive ouverte pluridisciplinaire HAL, est destinée au dépôt et à la diffusion de documents scientifiques de niveau recherche, publiés ou non, émanant des établissements d'enseignement et de recherche français ou étrangers, des laboratoires publics ou privés. 


\section{DNA Directed immobilization glycocluster array: applications and perspectives}

Yann Chevolot* ${ }^{1}$, Emmanuelle Laurenceau $^{1}$, Magali Phaner-Goutorbe ${ }^{1}$, Virginie Monnier ${ }^{1}$, Eliane Souteyrand ${ }^{1}$, Albert Meyer ${ }^{2}$, Thomas Géhin ${ }^{1}$, Jean-Jacques Vasseur ${ }^{2}$ and François Morvan*2.

1 Université de Lyon, Institut des Nanotechnologies de Lyon UMR CNRS 5270, Ecole Centrale de Lyon, 36 Avenue Guy de Collongue, 69134 Ecully Cedex, France.

2 Institut des Biomolécules Max Mousseron (IBMM), UMR 5247 CNRS - Université Montpellier 1 Université Montpellier 2, Place Eugène Bataillon, CC1704, 34095 Montpellier cedex 5, France.

* Corresponding authors

Yann Chevolot: Université de Lyon, Institut des Nanotechnologies de Lyon UMR CNRS 5270, Ecole Centrale de Lyon, 36 Avenue Guy de Collongue, 69134 Ecully Cedex, France.

Tel: +33/472 186 240, fax: +33/4 784335 93, e-mail: yann.chevolot@ec-lyon.fr

François Morvan: Institut des Biomolécules Max Mousseron (IBMM), UMR 5247 CNRS - Université Montpellier 1 - Université Montpellier 2, Place Eugène Bataillon, CC1704, 34095 Montpellier cedex 5, France

Tel.: +33/467 144 961; fax: +33/467 042 029; e-mail: morvan@univ-montp2.fr

The present review concerns the recent advances in DNA Directed Immobilization (DDI) based glycocluster array. The impact of glycan immobilization on subsequent interactions with protein are discussed and the consequent pros and cons of DDI-based glycocluster array are reviewed. Finally, application in the discovery of anti-pathogen molecules is illustrated by screening for galactose or fucose glycoclusters targeting two Pseudomonas aeruginosa virulence factors (PA-IL and PA-IIL).

Highlights

-We review advances in DNA Directed Immobilization based glycocluster array. Glycocluster array is a tool for anti-pathogen molecule discovery. Immobilization chemistry influences glycan/protein interactions in arrays. The dissociation constant $(\mathrm{Kd})$ and $\mathrm{IC}_{50}$ of glycoclusters targeting Pseudomonas aeruginosa virulence factors are determined with picomoles of molecules. 


\section{Introduction}

Glycoarrays, also known as glycan arrays or carbohydrate microarrays, emerged between the late 1990s and early 2000s [1-5]. The success of the Consortium for Functional Glycomics (CFG) microarray has demonstrated the power of this tool for basic research in glycobiology. Omics glycan arrays are aimed at determining the specificity of carbohydrate/protein interactions and as such need to have as many different structures as possible.

Glycan arrays are also powerful tools for therapeutic application to screen carbohydrate-derived drugs. In this case, microarrays should provide both qualitative and quantitative information using only minute amounts of material. However, quantitative information can be made more reliable if the surface densities of biologically active probes, mass transport and non-specific adsorption are well controlled.

The present review focuses on DNA-Directed Immobilization (DDI) based glycocluster microarrays and their applications for drug discovery, particularly glycoclusters targeting Pseudomonas aeruginosa (PA) virulence factors and more specifically the two soluble lectins Lec A (PA-IL) and Lec B (PA-IIL). Here, glycoclusters are defined as multivalent molecules bearing 2 to 10 carbohydrate residues (generally monosaccharides). While monovalent interactions between monosaccharides and lectins are generally weak, glycoclusters exploit the so-called 'glycoside cluster effect' to gain several orders of magnitude in affinity [6]. For a review of multivalent glycoconjugates as anti-pathogenic agents, see Bernadi et al. [7*].

The first section of the present paper is devoted to the influence of carbohydrate immobilization on lectin/carbohydrate interactions. The second section concerns the design of DDI-based glycocluster microarrays and their implementation as a tool to gain quantitative information. Section three concerns the synthesis of glycoclusters and their screening for PA virulence factors. The final section deals with the future development and application of DDI glycocluster arrays.

\section{Carbohydrate immobilization and their interactions with proteins}

We have elected not to discuss the different chemistries by which carbohydrates are immobilized, but focus our discussion on how the interactions between immobilized carbohydrates and proteins can be affected by surface chemistry considerations. These issues probably make simple comparison between different array formats difficult [8*]. Similarly, intra-slide comparisons can also be affected by surface chemistry, which can bias results.

The first obvious parameter is steric hindrance. Steric hindrance of immobilized molecules can emerge from the vicinity of the surface and/or from the vicinity of neighboring immobilized molecules. Although common sense would suggest using long spacer to extend the molecules away from the surface, several publications have demonstrated that such spacers can also lead to closer packing of surface molecules resulting in steric hindrance $[9,10]$.

Carbohydrate/lectin interactions can also be affected by molecular orientation, degree of freedom and other interface phenomena such as surface solvation. It is quite well documented that the water solvation layer plays a key role in biomolecule/biomolecule interactions [11] as well as at the 
solid/liquid interfaces, for example on protein adsorption [12]. Leung et al., using Sum Frequency Generation, demonstrated that water forms an ordered network extending from the bulk of a polyethylene oxide (PEO)-modified surface into the bulk of the solution $(1 \mathrm{~nm})$ and that this network is responsible for PEO antifouling properties [13]. It can therefore be imagined that interbiomolecular interactions which are affected by water solvation can also be affected by different water solvations at solid/liquid interfaces for different microarray formats. Concerning the orientation of immobilized molecules, Popplewell et al. demonstrated a difference in orientation between immobilized keratan sulfate and chondroitin sulfate oligosaccharides on hydrazine-modified surfaces, despite the use of the same chemistry [14]. More recently, Padler-Karavani et al. crosscompared interaction profiles for two different sialoglycan arrays with various sialic acid-recognizing proteins, and found different results for different arrays [ $\left.8^{*}\right]$. They suggested that different array formats lead to different presentations of the sialoglycan.

In monovalent interactions, carbohydrate binding proteins (lectins) exhibit high dissociation constants. However, lectins are multimers, bearing several carbohydrate recognition domains (CRDs). Hence, multiple contacts between a lectin and carbohydrates can lead to a very large increase in measured avidity, providing that the topology of the carbohydrate residues fits the topology of the lectin CRDs. This is the so-called 'glycoside cluster effect'. This enhancement is observed with multivalent ligands including glycoclusters, glycodendrimers and glycopolymers, but can also be observed on surfaces, typically cell surfaces, liposomes, nanoparticles [15] and glycan arrays $[16,17]$. Hereafter, this phenomenon will be called the 'surface glycoside cluster effect'. The extent of the surface glycoside cluster effect is a function of the distance between single carbohydrate residues on the surface. It can be correlated to the surface density of immobilized molecules if these are spread homogeneously over the surface. Using a mucin mimic array, Godula et al. [18*] demonstrated that glycan valency and organization were critical parameters affecting interaction in a panel of four lectins. Consequently, the relative surface density of each immobilized molecule should ideally be assessed.

In the case of carbohydrate-derived drugs, the interaction between the drug and the therapeutic target will occur freely in a solution involving the interaction of one drug molecule with one target molecule. Similarly, for drug screening assays on microarrays, the interaction between the immobilized carbohydrate drug and the target should mimic the interaction in solution as closely as possible. Therefore, the target should interact with only one immobilized drug and avoid any surface glycoside cluster effect.

\section{DDI glycocluster array}

DDI uses a DNA microarray as immobilization matrix: DNA conjugates are immobilized by hybridization with their complementary sequence on the DNA array. Peptide Nucleic Acid (PNA) conjugates have also been used [19]. DDI has been used to immobilize proteins and aptamers. Since an excellent review of DDI is available [20], we here focus on DDI of glycoclusters and the experimental determination of their $\mathrm{IC}_{50} \mathrm{~S}$ and dissociation constants (Kd) toward PA virulence factors. 


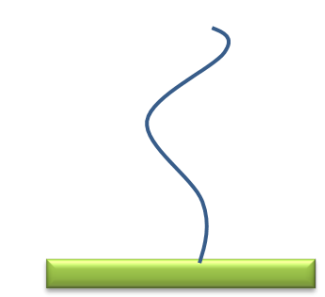

DNA microarray

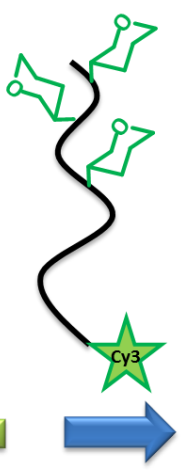

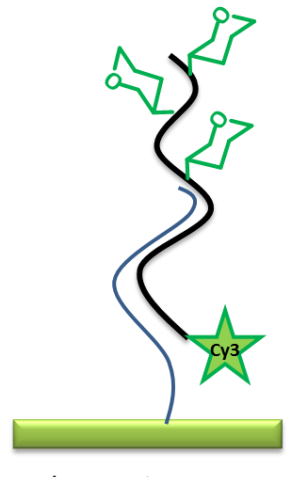

Glyco microarray
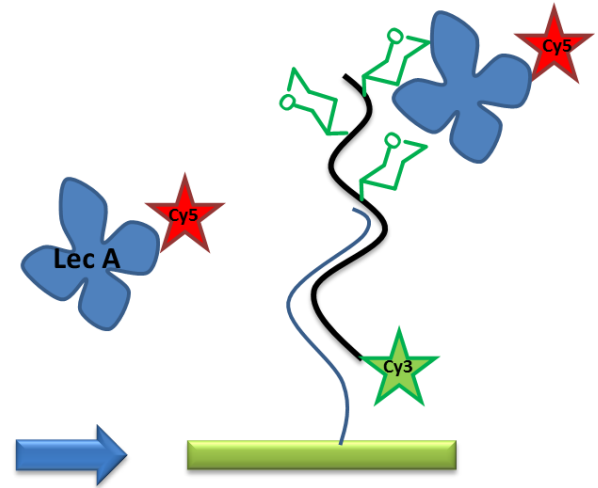

Figure 1: Principle of the DDI glycoarray. Immobilization of the Cy3-DNA-glycocluster followed by incubation with Cy5-labeled lectin and binding to the glycocluster moiety. Cy3 and Cy5: cyanine 3 and cyanine 5, respectively; Lec A: PA-IL.

In our system, the DNA immobilization matrix consists of a set of amino-linker (7-11 methylene) modified oligonucleotide sequences covalently immobilized by $\mathrm{N}$-hydroxysuccinimide-ester chemistry [21]. The DNA-glycocluster conjugates are labeled with a cyanine-3 fluorescent tag (Cy3) for immobilization quality control: the fluorescent $\mathrm{Cy} 3$ signal is related to DDI glycocluster surface density (see Figure 1) [22]. The bacterial lectins are labeled with cyanine 5 (Cy5) or an equivalent fluorescent tag such as Alexa 647: the fluorescent signal corresponds to surface lectin interacting with the glycocluster head.

The surface densities of hybridized ${ }^{32} \mathrm{P}$-labeled oligonucletotides were estimated to be in the $3 \times 10^{11}$ molecules $/ \mathrm{cm}^{2}$ range [23]. A similar DDI glycocluster surface density is expected. In this case, the average distance between two glycoclusters is approximately 50-60 nm. PA lectin, LecA (PA-IL), is a tetrameric galactose-binding lectin with a $7.0 \mathrm{~nm} \times 3.2 \mathrm{~nm} \times 1.9 \mathrm{~nm}$ cobblestone shape [24]. Its size is at least 7-fold smaller than the average distance between two immobilized molecules, so that the lectin can interact with only one glycocluster at a time. In other words, the observed glycoside cluster effects are related only to the multivalency of the molecules (see below) and not to a surface glycoside cluster effect. The resulting double-stranded DNA leads to a rigid arm, allowing better presentation of the glycocluster to the media; hence sensitivity is better than with covalent immobilization. [25]

Another advantage of DDI-based glycocluster arrays for drug discovery stems from the possibility of probing the lectin/glycocluster interactions in solution before addressing the whole lectin/glycocluster complex on the DNA microarray [22].

For multiplexed assays probing several lectin/glycocluster interactions in a single experiment, each glycocluster should carry a different oligonucleotide tag and each DNA tag should hybridize specifically with its complementary sequence in the DNA array. Additionally, the hybridization yield should be similar under similar experimental conditions to obtain similar surface densities. However, differences can be corrected taking into account the Cy3 signal from the hybridized glycocluster. Thus, one of the challenges with DDI is to design a set of DNA sequences allowing multiplexed assay. For this, a set of sequences known to have similar hybridization yields on DNA microarray was used. For each selected oligonucleotide, new sequences were generated by randomization of triplex 
segment positions. Specificity and free hybridization enthalpy were evaluated in-silico using DINAmelt server [26]. Following the in-silico screening, the remaining sequences were assessed experimentally [21]. Nevertheless, the design of larger sequence sets (above 50) is one of the main hurdles in DDI.

To determine $\mathrm{IC}_{50}$ and $\mathrm{Kd}$, biomolecular interactions need to be carried out under different experimental conditions. For $\mathrm{IC}_{50} \mathrm{~S}$, inhibition curves are obtained with increasing concentrations of a reference inhibitor; the $\mathrm{IC}_{50}$ value corresponds to the concentration displacing $50 \%$ of the lectin bound to the immobilized glycocluster $[25,27]$. For Kds, isotherms are obtained with increasing concentrations of lectins, followed by linear regression [28*]. Discrete data points are obtained using glass microscope slides bearing 40 etched microwells. The DNA immobilization matrix is printed at the bottom of each well and each well corresponds to a given concentration of inhibitor or lectin [28*].

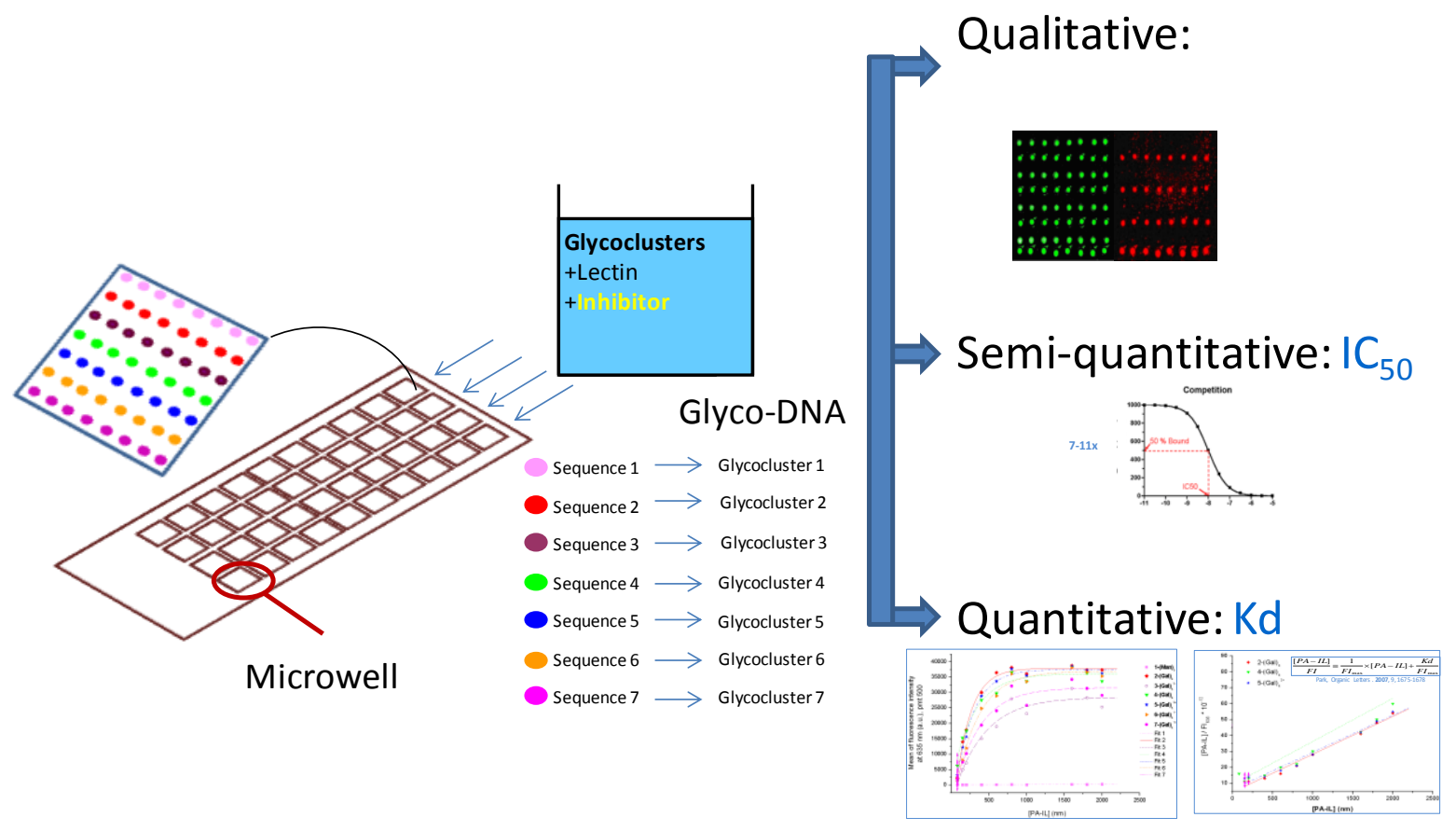

Figure 2: Diagram of the DDI glycocluster array. The DNA immobilization matrix is printed at the bottom of each well. To determine $\mathrm{IC}_{50}$, glycoclusters and the targeted $P A$ lectin at a given concentration are incubated with increasing concentrations of inhibitor. Each microwell corresponds to a given concentration of inhibitor. To determine $\mathrm{Kd}$, glycoclusters are incubated with increasing concentrations of lectin. Each microwell corresponds to a given concentration of lectin. $\mathrm{IC}_{50}$ : half maximal inhibitory concentration; Kd: dissociation constant.

\section{Screening glycoclusters targeting $P A$ virulence factors}

$P A$ is a Gram-negative opportunistic pathogen. It is one of the three most prevalent nosocomial bacterial pathogens (10-30\% of nosocomial infections) and the major cause of morbidity and mortality in cystic fibrosis. The emergence of resistant strains and biofilm formation seems to give the bacterium a selective advantage over antibiotic therapy. A promising approach is to inhibit the virulence factors of $P A$, and particularly the adhesins and lectins involved in bacterial adhesion and 
biofilm formation. Our work has so far focused on Lec A (PA-IL) and Lec B (PA-IIL), two tetrameric lectins targeting $\mathrm{D}$-galactose and L-fucose residues, respectively. High-affinity ligands of these two lectins can be obtained, with multivalent ligands taking advantage of the glycoside cluster effect [15,29-34]. Overall avidity depends on the topology, linker arm and carbohydrate structure [35]. To our knowledge, there are as yet no means of predicting optimal structures. Here, glycoclusters were synthesized at a 10 nanomole scale and screened on DDI array.

DNA glycoclusters are synthesized using a combination of automated DNA solid supported chemistry and copper catalyzed alkyne azide cycloaddition "click" chemistry [36-38]. To this end, building blocks are assembled in a "Lego" based approach leading to poly alkynes or poly azide scaffold with different topologies according nucleic acid chemistry on a DNA synthesizer. Then, azido or alkynyl modified glycosides are introduced by click chemistry. The resulting glycoclusters with a linear scaffold exhibit different linkers L3 between two phosphoramidate diesters linkages bearing the carbohydrate moiety. Carbohydrate-centered glycoclusyters were synthesized from different monohexoses (mannose, galactose or glucose) where each hydroxyl allows the introduction of the carbohydrate moiety through different linkers L2 (Figure 3). The linkers L1 and L2 can be modulated leading to an even greater diversity of topology.

Furthermore, the physico-chemical properties of the molecules including hydrophilicity, lipophilicity, aromaticity and charge can be monitored using different linkers L1, L2 and L3. For example, amino groups can be introduced in the scaffold. At pH 7.2, these amines remain protonated introducing a positive charge in the molecule. Finally, the number of carbohydrate epitopes, can be varied by controlling the number of building blocks introduced in the scaffold. $[27,34,39,40]$.

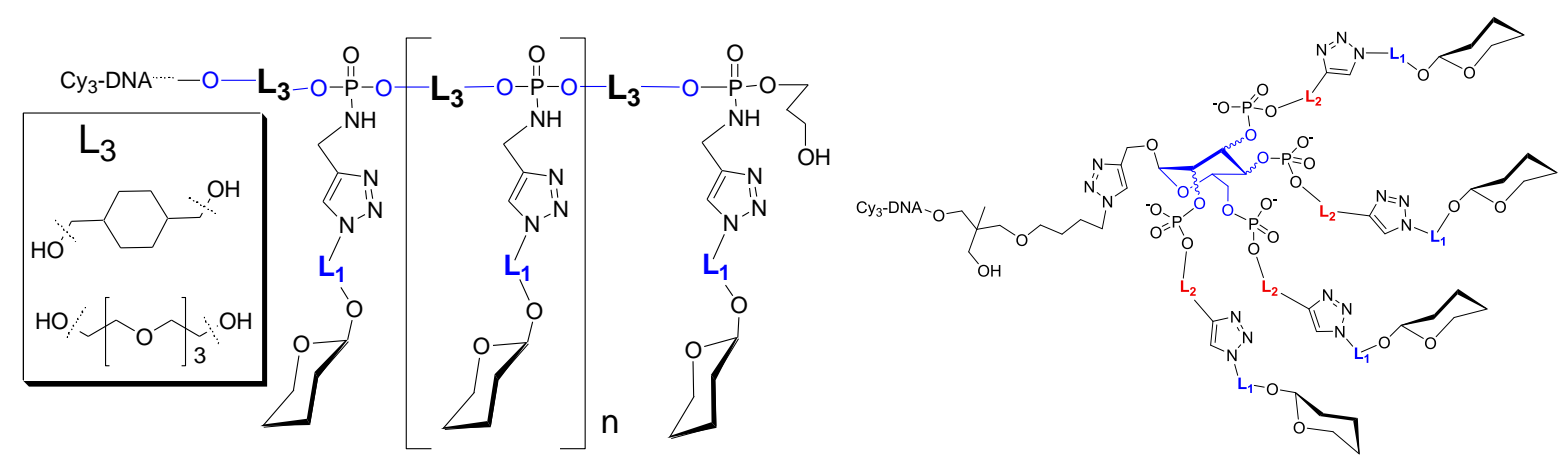

Figure 3: General structures of glycoclusters, based on a) H-phosphonate chemistry and b) phosphoramidite chemistry. L: linker. 


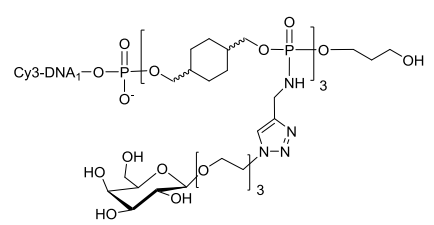

Go

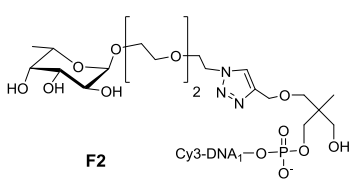

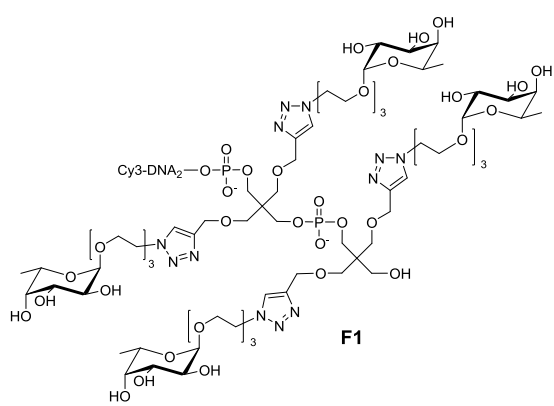

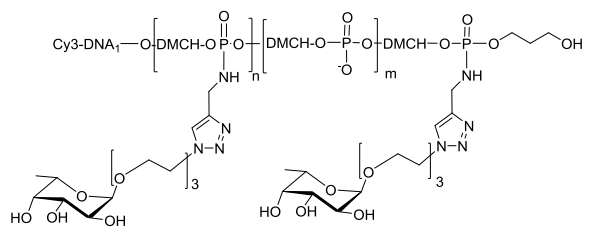

F3 a: $n=1, m=0$

F4 b: $n=2, m=0$
F5 c: $n=3, m=0$

F6 d: $n=4, m=0$ F7 $\mathrm{m}: \mathrm{n}=2, \mathrm{~m}=1$
$\mathrm{~m}=1$

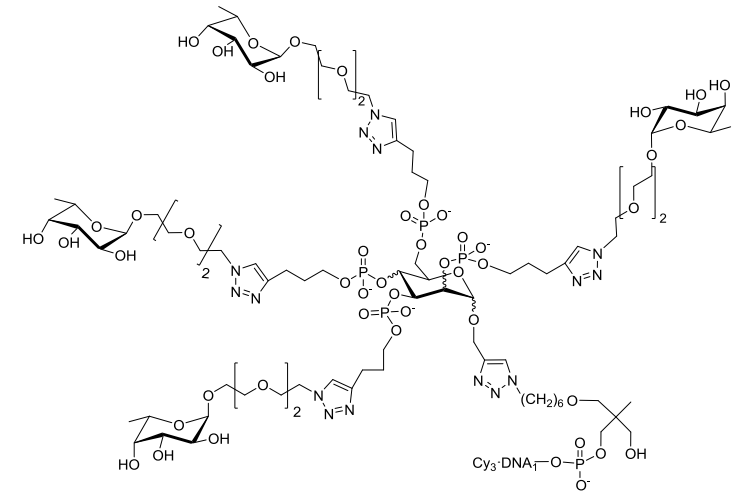

F8 : Man
F9 : Gal
F10: Glc

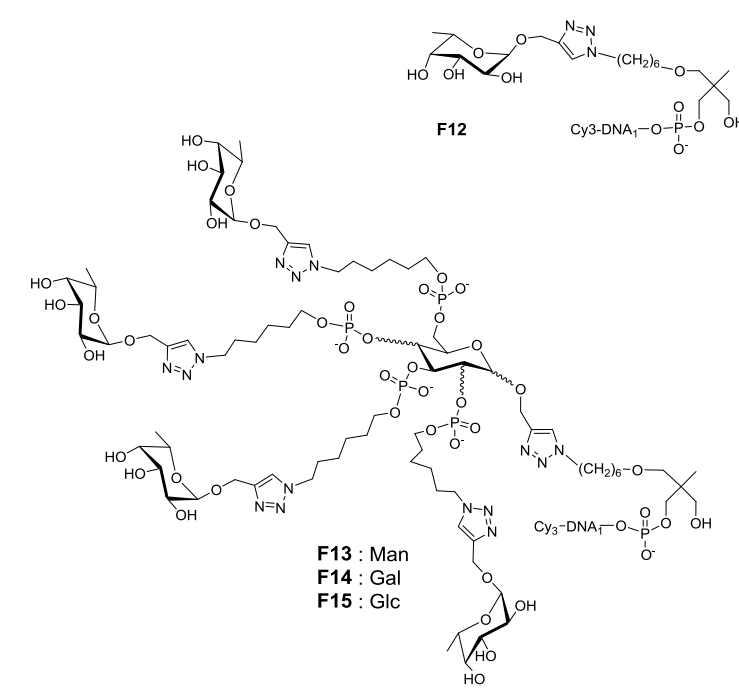

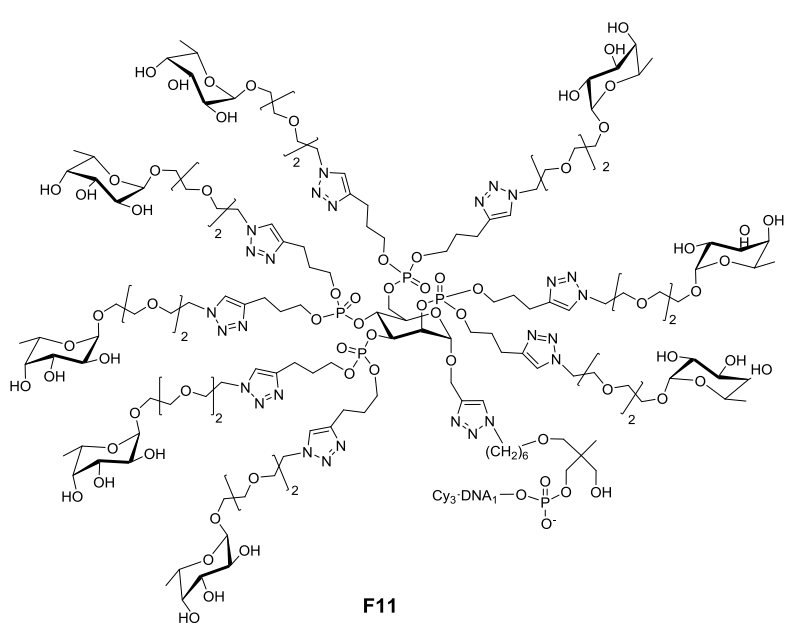
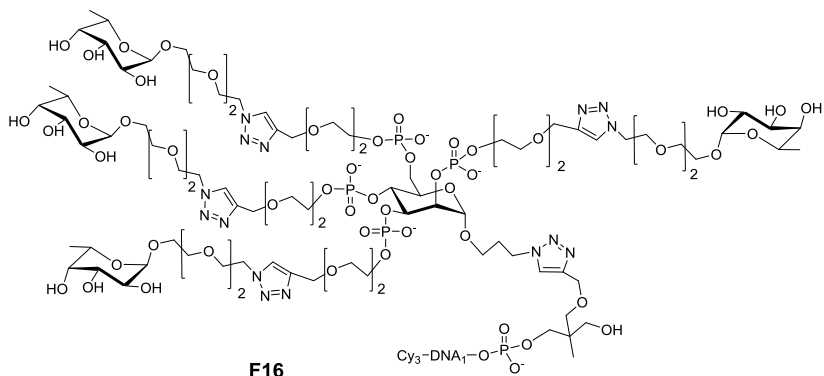

Figure 4: Overview of the different fucose clusters. Reprinted with permission from [41]. Copyright 2012, American Chemical Society. Man: mannose; Gal: galactose; Glc: glucose. 
In a recent report, the binding of 16 different fucosylated clusters targeting PA-IIL was evaluated. The impact of number of fucose residues (1 to 8), topology (linear, antenna or crown), linker arm length (13 to 21 atoms) and position of the triazole ring from the anomeric carbon were evaluated (Figure 4). Topology was varied using various hexose-centered (glucose, mannose, and galactose, Figure 4 F8-F11 F13-F16), dimethylcyclohexane (DMCH, linear topology, Figure 4 F3-F7) or bis-pentaerythrityl scaffolds (antenna topology, Figure 4 F1). The various hexose cores allowed the spatial orientation to be probed. Direct fluorescent read-out and on-chip $\mathrm{IC}_{50}$ assay suggested that PA-IL bound preferentially to linear antennae or mannose-centered scaffolds. Binding also increased with increasing linker arm length and increasing number of fucose residues, but no chelate effects were observed [41].

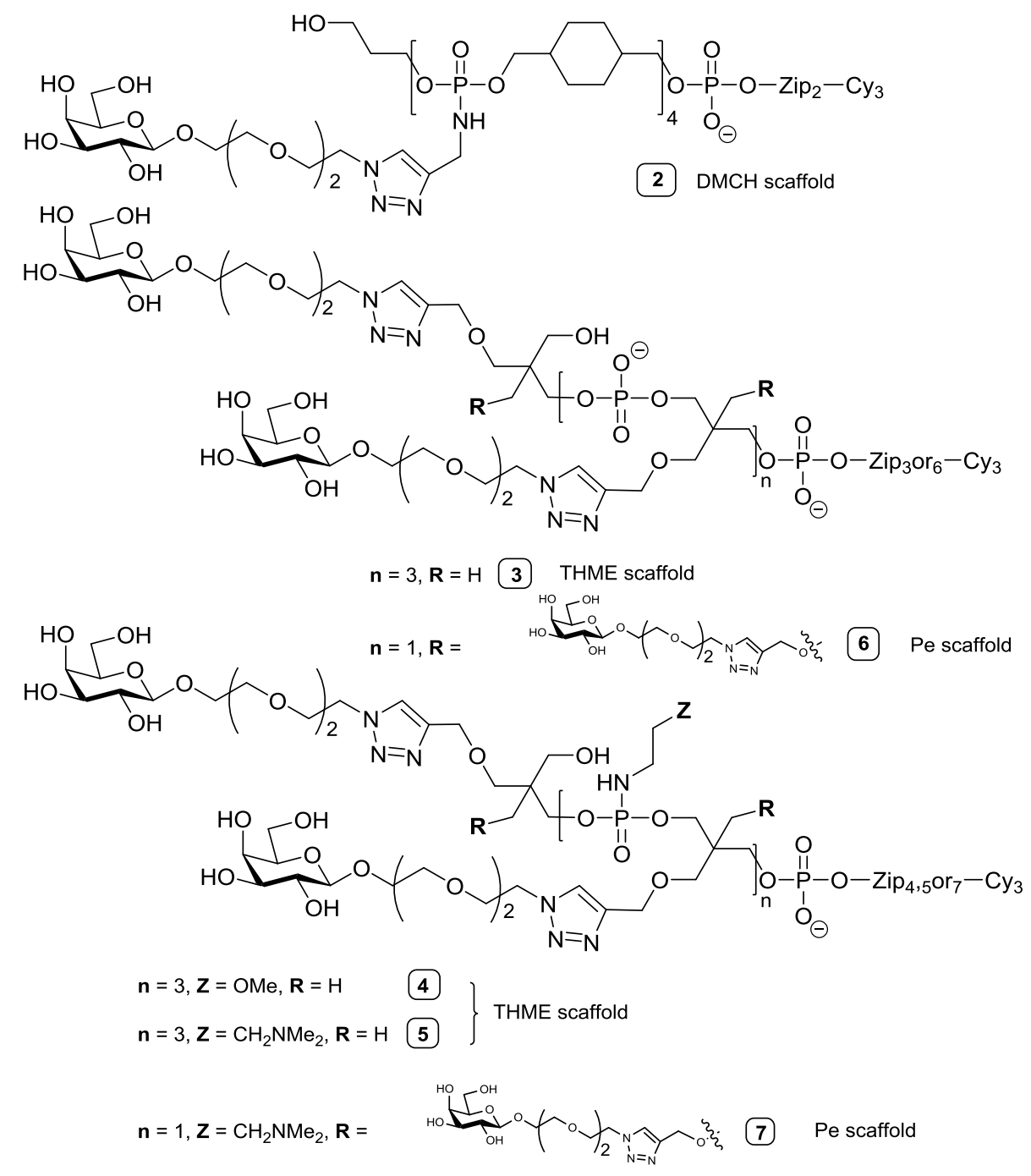

Figure 5: General structure of tetrameric galactose clusters.

In a multiplexed system made possible by using different DNA tags linked to each glycocluster, Goudot et al., using a DDI glycocluster array, measured the $\mathrm{IC}_{50} \mathrm{~s}$ and Kds of PA-IL toward 6 tetrameric galactoclusters bearing one or three positive or negative charges or else neutral toward PA-IL (Figure 
5) $\left[28^{*}\right]$. The direct fluorescent read-out, $\mathrm{IC}_{50}$ and $\mathrm{Kd}$ experiments demonstrated that, for a given family, positively-charged scaffolds enhanced PA-IL binding while negatively-charged scaffolds hindered it. These attractive or repulsive electrostatic interactions between lectin and galactocluster moieties probably originated from the negative charge of PA-IL at pH 7.4. The best affinity was observed for the neutral galactocluster built on a $\mathrm{DMCH}$ scaffold. Direct fluorescent scanning, $\mathrm{IC}_{50}$ and $\mathrm{Kd}$ provided almost identical affinity distributions. It is noteworthy that only 40 picomoles of each DNA-glycocluster were needed for these measurements.

In order to target both PA-IL and PA-IIL, a heteroglycocluster exhibiting two mannose-centered cores, carrying respectively four galactose and four fucose residues on the same structure, was synthesized. Their IC $\mathrm{I}_{50}$ for PA-IL and for PA-IIL were compared to the corresponding homoclusters (i.e., mannosecentered tetragalactose or tetrafucose). Although binding was slightly decreased, dual binding to both lectins was shown to be achievable [42*].

\section{Conclusions and perspectives}

In summary, DDI glycocluster arrays allow measurement of the $\mathrm{Kd}$ and $\mathrm{IC}_{50}$ values of multivalent molecules driven drug targeting bacterial lectin. These experimental measurements were not performed on a real-time basis (fluorescence was measured after the final washing step). An improvement would be to combine a microfluid system with real-time detection such as Quartz Crystal Microbalance or Surface Plasmon imaging. Thus, deeper thermodynamic (enthalpy and entropy) and kinetic insight could be gained, measuring the equilibrium constant at different temperatures [43]. Furthermore, such a microsystem combining DDI and Van't Hoff-based assay would allow the entropic cost of heterogeneous-phase biomolecular interaction to be compared to homogeneous-phase interaction. Finally, it is predicted that integration into microfluid systems should increase screening pace while decreasing material consumption.

Using DDI-based glycocluster arrays, we were able to identify high-affinity ligands targeting PA-IL and PA-IIL involved in PA virulence factors. Synthesis has been scaled up and their anti-pathogen activity is currently being investigated in cell culture. Lectin PA-IL/glycocluster complexes have also been investigated by Atomic Force Microscope (AFM) at a nanometric scale and showed nanostructural arrangements in filaments [24].

In future, AFM will be used in its spectroscopic mode to measure the interaction force between lectin and several glycoclusters and also between bacterium and cell. Theses local measurements will be compared to Kd values.

Another interesting future development in the field of glycoclusters concerns the use of nanoparticles functionalized with glycoclusters or carbohydrates (also called 'glyconanoparticles' [44]). They comprise an inorganic core exhibiting one or several physical properties (such as fluorescent [45], plasmonic [46] and magnetic properties [47]). For example, glyconanoparticles combining fluorescent and magnetic properties have been reported for in-vivo imaging of $B$ lymphocytes: their fluorescent properties enabled fluorescent microscopy while their magnetic properties made them good a T2 contrast agent for MRI [47]. Tong et al. reported the development of a glyconanoparticle microarray, in which carbohydrate-modified silica nanoparticles are printed 
and immobilized on a polymeric matrix by photochemistry; the fluorescent signal was enhanced compared to conventional microarray. [48]. Glyconanoparticles can also be used in biomedical applications, as reported by Reynolds et al.: they showed enhanced binding toward lectins thanks to the surface cluster effect and could be exploited as anti-pathogens [15]. For example, magnetic glycocluster nanoparticles could also be used to catch and isolate thanks to the magnetic properties and to identify new lectins from complex media.

\section{Acknowledgements}

We thank Dr C. Bouillon, Dr S. Cecioni, Dr X. Chen, Dr J. P. Cloarec, Dr B. Gerland, Alice Goudot, Dr A. Imberty, Dr J. Lietard, C. Ligeour, Pr A. Marra, Dr L. Moni, Dr G. Pourceau, Dr J. P. Praly, S. Rouanet, A. Thiam, Dr O. Vidal, Dr S. Vidal and Dr J. Zhang, who greatly contributed to the success of this project. The study was financially supported by the CNRS interdisciplinary program "Interface Physique Chimie Biologie: Soutien à la prise de risque", ANR-08-BLAN-0114-01, Lyon Biopole, Eurobiomed, ANR-12-BSV5-0020-01, Vaincre la Mucoviscidose, CNRS ANR-12-RPIB-003-04, the LanguedocRoussillon Regional Council and Montpellier 2 University. F. M. is member of Inserm. Plateforme NanoLyon is acknowledged for technical support. 
1. Blixt O, Head S, Mondala T, Scanlan C, Huflejt M, E., Alvarez R, Bryan M, C., Fazio F, Calarese D, Stevens J, et al.: Printed covalent glycan array for ligand profiling of diverse glycan binding proteins. Proc. Natl. Acad. Sci. U. S. A. 2004, 10:17033-17038.

2. Fukui S, Feizi T, Galustian C, Lawson AM, Chai W: Oligosaccharide microarrays for high-throughput detection and specificity assignments of carbohydrate-protein interactions. Nat. Biotechnol. 2002, 20:1011-1017.

3. Leonard D, Chevolot Y, Bucher O, Haenni W, Sigrist H, Mathieu HJ: ToF-SIMS and XPS study of photoactivatable reagents designed for surface glycoengineering - Part 2. N-[m-(3(trifluoromethyl)diazirine-3-yl)phenyl]-4-(-3-thio(-1-D-galactopyrannosyl)maleimidyl)butyramide (MAD-Gal) on diamond. Surf. Interface Anal. 1998, 26:793-799.

4. Wang D, Liu S, Trummer BJ, Deng C, Wang A: Carbohydrate Microarrays for the Recognition of Cross-reactive Molecular Markers of Microbes and Host Cells. Nat. Biotechnol. 2002, 20:275-281.

5. Park S, Gildersleeve JC, Blixt O, Shin I: Carbohydrate microarrays. Chem. Soc. Rev. 2013, 42:43104326.

6. Lee YC, Lee RT: Carbohydrate-Protein Interactions: Basis of Glycobiology. Acc. Chem. Res. 1995, 28:321-327.

7*. Bernardi A, Jimenez-Barbero J, Casnati A, De Castro C, Darbre T, Fieschi F, Finne J, Funken H, Jaeger K-E, Lahmann M, et al.: Multivalent glycoconjugates as anti-pathogenic agents. Chem. Soc. Rev. 2013, 42:4709-4727.

Good overview of the use of multivalent ligand targeting viruses, bacterial toxin, bacterial adhesion factors or as vaccine approach.

8*. Padler-Karavani V, Song X, Yu H, Hurtado-Ziola N, Huang S, Muthana S, Chokhawala HA, Cheng J, Verhagen A, Langereis MA, et al.: Cross-comparison of Protein Recognition of Sialic Acid Diversity on Two Novel Sialoglycan Microarrays. J. Biol. Chem. 2012, 287:22593-22608.

In this publication two sialoglycan microarray formats are compared. It underlines how array format can affect binding results.

9. Grubor N, M., Shinar R, Jankowiak R, Porter M, D., Small G, J.: Novel biosensor chip for simultaneous detection of DNA-carcinogen adducts with low temperature fluorescence. Biosens. Bioelectron. 2004, 19:547-556.

10. Miura $Y$, Sasao $Y$, Dohi H, Nishida $Y$, Kobayashi K: Self-assembled monolayers of globotriaosylceramide (Gb3) mimics: surface-specific affinity with shiga toxins. Anal. Biochem. 2002, 310:27-35.

11. Patel AJ, Varilly P, Jamadagni SN, Hagan MF, Chandler D, Garde S: Sitting at the Edge: How Biomolecules use Hydrophobicity to Tune Their Interactions and Function. J. Phys. Chem. $B$ 2012, 116:2498-2503. 
12. Ratner BD, Hoffman AS, Schoen FJ, Lemons JE (Ed): Biomaterials Science. An Introduction to Materials in Medicine. Third Edition Oxford: Academic Press; 2013.

13. Leung BO, Yang Z, Wu SSH, Chou KC: Role of Interfacial Water on Protein Adsorption at CrossLinked Polyethylene Oxide Interfaces. Langmuir 2013, 28:5724-5728.

14. Popplewell JF, Swann MJ, Ahmed Y, Turnbull JE, Fernig DG: Fabrication of Carbohydrate Surfaces by Using Nonderivatised Oligosaccharides, and their Application to Measuring the Assembly of Sugar-Protein Complexes. ChemBioChem 2009, 10:1218-1226.

15. Reynolds M, Marradi M, Imberty A, Penades S, Perez S: Multivalent Gold Glycoclusters: High Affinity Molecular Recognition by Bacterial Lectin PA-IL. Chem. Eur. J. 2012, 18:4264-4273.

16. Rillahan CD, Paulson JC: Glycan Microarrays for Decoding the Glycome. Annu. Rev. Biochem. 2011, 80:797-823.

17. Liang C-H, Wang S-K, Lin C-W, Wang C-C, Wong C-H, Wu C-Y: Effects of Neighboring Glycans on Antibody-Carbohydrate Interaction. Angew. Chem., Int. Ed. Engl. 2011, 50:1608-1612.

18*. Godula K, Bertozzi CR: Density Variant Glycan Microarray for Evaluating Cross-Linking of Mucin-like Glycoconjugates by Lectins. J. Am. Chem. Soc. 2012, 134:15732-15742.

The effect of glycan density on the dissociation constant is well discussed.

19. Daguer J-P, Ciobanu M, Barluenga S, Winssinger N: Discovery of an entropically-driven small molecule streptavidin binder from nucleic acid-encoded libraries. Org. Biomol. Chem. 2012, 10:1502-1505.

20. Niemeyer CM: Semisynthetic DNA-Protein Conjugates for Biosensing and Nanofabrication. Angew. Chem., Int. Ed. Engl. 2010, 49:2-19.

21. Chevolot Y, Zhang J, Meyer A, Goudot A, Rouanet S, Vidal S, Pourceau G, Cloarec J-P, Praly J-P, Souteyrand $E$, et al.: Multiplexed binding determination of seven glycoconjugates for Pseudomonas aeruginosa Lectin I (PA-IL) using a DNA-based carbohydrate microarray. ChemComm 2011, 47:8826-8828.

22. Zhang J, Pourceau G, Meyer A, Vidal S, Praly JP, Souteyrand E, Vasseur JJ, Morvan F, Chevolot Y: Specific recognition of lectins by oligonucleotide glycoconjugates and sorting on a DNA microarray. Chem Comm 2009:6795-6797.

23. Dugas V, Depret G, Chevalier B, Nesme X, Souteyrand E: Immobilization of single-stranded DNA fragments to solid surfaces and their repeatable specific hybridization: covalent binding or adsorption? Sens. Actuators, B 2004, 101:112-121.

24. Sicard D, Cecioni S, lazykov M, Chevolot $Y$, Matthews SE, Praly J-P, Souteyrand E, Imberty A, Vidal $S$, Phaner-Goutorbe M: AFM investigation of Pseudomonas aeruginosa lectin LecA (PA-IL) filaments induced by multivalent glycoclusters. ChemComm 2011, 47:9483-9485.

25. Zhang J, Pourceau G, Meyer A, Vidal S, Praly J-P, Souteyrand E, Vasseur J-J, Morvan F, Chevolot Y: DNA-directed immobilisation of glycomimetics for glycoarrays application: Comparison with covalent immobilisation, and development of an on-chip IC50 measurement assay. Biosens. Bioelectron. 2009, 24:2515-2521. 
26. Markham NR, Zuker M: DINAMelt web server for nucleic acid melting prediction. Nucleic Acids Res. 2005, 33:W577-W581.

http://mfold.rna.albany.edu/?q=dinamelt

27. Moni L, Pourceau G, Zhang J, Meyer A, Vidal S, Souteyrand E, Dondoni A, Morvan F, Chevolot Y, Vasseur J-J, et al.: Design of Triazole-Tethered Glycoclusters Exhibiting Three Different Spatial Arrangements and Comparative Study of Their Affinity for PAIL and RCA 120 Using a DNA-Based Glycoarray. ChemBioChem 2009, 10:1369-1378.

28*. Goudot A, Pourceau G, Meyer A, Gehin T, Vidal S, Vasseur J-J, Morvan F, Souteyrand E, Chevolot $Y$ : Quantitative analysis (K(d) and IC(50)) of glycoconjugates interactions with a bacterial lectin on a carbohydrate microarray with DNA Direct Immobilization (DDI). Biosens. Bioelectron. 2013, 40:153-160.

A unique DDI platform is described for the multiplexed determination of $\mathrm{Kd}$ and $\mathrm{IC}_{50}$ of glycocuster toward PA-IL with only 40 picomoles of glycoclusters.

29. Chabre YM, Giguere D, Blanchard B, Rodrigue J, Rocheleau S, Neault M, Rauthu S, Papadopoulos A, Arnold AA, Imberty A, et al.: Combining Glycomimetic and Multivalent Strategies toward Designing Potent Bacterial Lectin Inhibitors. Chem. Eur. J. 2011, 17:6545-6562.

30. Consoli GML, Granata G, Cafiso V, Stefani S, Geraci C: Multivalent calixarene-based C-fucosyl derivative: a new Pseudomonas aeruginosa biofilm inhibitor. Tetrahedron Lett. 2011, 52:5831-5834.

31. Kadam RU, Bergmann M, Hurley M, Garg D, Cacciarini M, Swiderska MA, Nativi C, Sattler M, Smyth AR, Williams P, et al.: A Glycopeptide Dendrimer Inhibitor of the Galactose-Specific Lectin LecA and of Pseudomonas aeruginosa Biofilms. Angew. Chem. Int. Ed. Engl. 2011, 50:10631-10635.

32. Pertici F, Pieters RJ: Potent divalent inhibitors with rigid glucose click spacers for Pseudomonas aeruginosa lectin LecA. ChemComm 2012, 48:4008-4010.

33. Reymond J-L, Darbre T: Peptide and glycopeptide dendrimer apple trees as enzyme models and for biomedical applications. Org. Biomol. Chem. 2012, 10:1483-1492.

34. Morvan F, Meyer A, Jochum A, Sabin C, Chevolot Y, Imberty A, Praly JP, Vasseur JJ, Souteyrand E, Vidal S: Fucosylated pentaerythrityl phosphodiester oligomers (PePOs): Automated synthesis of DNA-Based glycoclusters and binding to Pseudomonas aeruginosa lectin (PAIIL). Bioconjug. Chem. 2007, 18:1637-1643.

35. Cecioni S, Praly J-P, Matthews SE, Wimmerova M, Imberty A, Vidal S: Rational Design and Synthesis of Optimized Glycoclusters for Multivalent Lectin-Carbohydrate Interactions: Influence of the Linker Arm. Chem. Eur. J. 2012, 18:6250-6263.

36. Chevolot Y, Bouillon C, Vidal S, Morvan F, Meyer A, Cloarec JP, Jochum A, Praly JP, Vasseur JJ, Souteyrand E: DNA-based carbohydrate biochips: A platform for surface glyco-engineering. Angew. Chem., Int. Ed. Engl. 2007, 46:2398-2402.

37. Morvan F, Meyer A, Pourceau G, Vidal S, Chevolot Y, Souteyrand E, Vasseur JJ: Click chemistry and Oligonucleotides: How a simple reaction can do so much. Nucleic Acids Symp. Ser. 2008, 52:47-49. 
38. Spinelli N, Defrancq E, Morvan F: Glycoclusters on oligonucleotide and PNA scaffolds: synthesis and applications. Chem. Soc. Rev. 2013, 42:4557-4573.

39. Morvan F, Vidal S, Souteyrand E, Chevolot Y, Vasseur J-J: DNA glycoclusters and DNA-based carbohydrate microarrays: From design to applications. R. Soc. Chem. Adv. 2012, 2:1204312068.

40. Pourceau G, Meyer A, Chevolot Y, Souteyrand E, Vasseur JJ, Morvan F: Oligonucleotide Carbohydrate-Centered Galactosyl Cluster Conjugates Synthesized by Click and Phosphoramidite Chemistries. Bioconjug. Chem. 2010, 21:1520-1529.

41. Gerland B, Goudot A, Pourceau G, Meyer A, Dugas V, Cecioni S, Vidal S, Souteyrand E, Vasseur J-J, Chevolot $Y$, et al.: Synthesis of a Library of Fucosylated Glycoclusters and Determination of their Binding toward Pseudomonas aeruginosa Lectin B (PA-IIL) Using a DNA-Based Carbohydrate Microarray. Bioconjug. Chem. 2012, 23:1534-1547.

42*. Gerland B, Goudot A, Pourceau G, Meyer A, Vidal S, Souteyrand E, Vasseur J-J, Chevolot Y, Morvan F: Synthesis of Homo- and Heterofunctionalized Glycoclusters and Binding to Pseudomonas aeruginosa Lectins PA-IL and PA-IIL. J. Org. Chem. 2012, 77:7620-7626.

This paper reports on the synthesis of a fucose/galactose hetero-cluster. More and more authors are suggesting that heterogeneous glycan should enhance affinity by mimicking natural complex presentation (see references 8 and 17).

43. Fuchs J, Fiche JB, Buhot A, Calemczuk R, Livache T: Salt Concentration Effects on Equilibrium Melting Curves from DNA Microarrays. Biophys. J. 2010, 99:1886-1895.

44. Garcia I, Gallo J, Genicio N, Padro D, Penades S: Magnetic Glyconanoparticles as a Versatile Platform for Selective Immunolabeling and Imaging of Cells. Bioconjug. Chem. 2011, 22:264273.

45. Bogdan N, Vetrone F, Roy R, Capobianco JA: Carbohydrate-coated lanthanide-doped upconverting nanoparticles for lectin recognition. J. Mater. Chem. 2011, 20:7543-7550.

46. Tsutsumi H, Ohkusa H, Park H, Takahashi T, Yuasa H, Mihara H: Gold nanoparticles conjugated with monosaccharide-modified peptide for lectin detection. Bioorg. Med. Chem. Lett. 2012, 22:6825-6827.

47. Gallo J, Garcia I, Genicio N, Padro D, Penades S: Specific labelling of cell populations in blood with targeted immuno-fluorescent/magnetic glyconanoparticles. Biomaterials 2011, 32:9818-9825.

48. Tong Q, Wang X, Wang H, Kubo T, Yan M: Fabrication of Glyconanoparticle Microarrays. Anal. Chem. 2012, 84:3049-3052. 


\section{Figure Captions}

Figure 1: Principle of the DDI glycoarray. Immobilization of the Cy3-DNA-glycocluster followed by incubation with Cy5-labeled lectin and binding to the glycocluster moiety. Cy3 and Cy5: cyanine 3 and cyanine 5, respectively; Lec A: PA-IL.

Figure 2: Diagram of the DDI glycocluster array. The DNA immobilization matrix is printed at the bottom of each well. To determine $\mathrm{IC}_{50}$, glycoclusters and the targeted $P A$ lectin at a given concentration are incubated with increasing concentrations of inhibitor. Each microwell corresponds to a given concentration of inhibitor. To determine $\mathrm{Kd}$, glycoclusters are incubated with increasing concentrations of lectin. Each microwell corresponds to a given concentration of lectin. $\mathrm{IC}_{50}$ : half maximal inhibitory concentration; Kd: dissociation constant.

Figure 3: General structures of glycoclusters, based on a) H-phosphonate chemistry and b) phosphoramidite chemistry. L: linker.

Figure 4: Overview of the different fucose clusters. Reprinted with permission from [41]. Copyright 2012, American Chemical Society. Man: mannose; Gal: galactose; Glc: glucose..

Figure 5: General structure of tetrameric galactose clusters. 